\title{
Crystal engineering of multicomponent crystal forms of antituberculosis drugs
}

Javier Ellena ${ }^{1}$, Luan Farinelli Diniz ${ }^{1}$, cristiane cabral de Melo ${ }^{1}$, Paulo de Sousa CARVALHO JR. ${ }^{1}$

${ }^{1}$ São Carlos Institute Of Physics - University Of São Paulo, SÃo Carlos, Brazil E-mail: javiere@ifsc.usp.br

Tuberculosis (TB) is one of the major causes of mortality in developing countries. Its high incidence and prevalence turns TB a global health problem. The TB treatment is based on the use of drugs as a fixed-dose combination (FDC) tablet which can simplify the TB treatment. However, one of the main concerns about the use of anti-TB drugs lies in the high hygroscopicity of Ethambutol (ETB) and limited solubility of Ethionamide (ETH). The development of multicomponent crystal forms, e.g. salts and cocrystals, represents an important branch of pharmaceutical sciences as alternative route to improve drug's physicochemical properties (aqueous solubility, hygroscopicity and thermal stability). Salt or cocrystal formation is a process strictly governed by the acidity/basicity of the ionizable groups in the drug and in the salt coformers. In general, pharmaceutical acceptable strong acids are used to protonate a basic drug, such as ETB and ETH and thereby convert it into salts. For ionizable drugs, salt formation is still the most effective low-cost method and consequently the preferential one to increase the low solubility and bioavailability of the parent drug. Based on the crystal engineering approach, we developed novel pharmaceutical salts of Isoniazid (INH), ETH and ETB anti-TB drugs. These salts were crystal engineered and supramolecular synthesized using a series of inorganic and carboxylic acids formers (maleic, nitric, sulfuric and oxalic) in order to overcome the undesirable effects of these drugs. The new salts obtained were study by single crystal $X$-ray diffraction as well as thermal and spectroscopy analysis. These studies showed that the assembly of ETH maleate salt is dominated by a cyclic tetramer arrangement where two ETH+ cations are alternately linked to two counterions. ETH nitrate crystallized with four independent ionic pairs in the asymmetric unit being the first ETH structure with $Z^{\prime}>1$ reported. Each ionic pair is stabilized by a strong pyridinium...NO3- H-bond. Solubility studies show that ETH nitrate salt is about $240-$ fold more soluble than ETH commercial API. In the INH sulfate salt, the INH+ cations form a rather unexpected $\mathrm{R}\left(\mathrm{2}^{\wedge} 2\right)(10)$ homodimers. The sulfate anions, in turn, bridge these homodimers into a $1-D$ chain via a $R\left(2^{\wedge} 2\right)(10)$ motif formed by pyridinium...SO4- H-bonds. Due to the presence of a much stronger anion- $\mathrm{INH}+\mathrm{H}$-bonds, this salt presents a high melting point (204 oC) when compared than INH parent form (m.p $170 \mathrm{oC}$ ). Analysis of the crystal structure and packing of ETB oxalate salt revealed that min this case the ion-pairs (ETB+/OXA-) are stabilized by the expected $\mathrm{NH}+\ldots \mathrm{COO}-\mathrm{synthons}$. Hygroscopicity tests of the ETB oxalate salt showed that this salt is non-hygroscopic making a suitable candidate for the anti-TB multiple-drug therapy formulation.

Keywords: Antituberculosis Drugs, fixed-dose combination, hygroscopicity 